\title{
Black hole attractors and the topological string
}

\section{Citation}

Ooguri, Hirosi, Andrew Strominger, and Cumrun Vafa. 2004. "Black Hole Attractors and the Topological String." Physical Review D 70 (10). https://doi.org/10.1103/physrevd.70.106007.

\section{Permanent link}

http://nrs.harvard.edu/urn-3:HUL.InstRepos:41385097

\section{Terms of Use}

This article was downloaded from Harvard University's DASH repository, and is made available under the terms and conditions applicable to Other Posted Material, as set forth at http:// nrs.harvard.edu/urn-3:HUL.InstRepos:dash.current.terms-of-use\#LAA

\section{Share Your Story}

The Harvard community has made this article openly available.

Please share how this access benefits you. Submit a story.

Accessibility 
hep-th/0405146

HUTP-04/A020, CALT-68-2501

\title{
Black Hole Attractors and the Topological String
}

\author{
Hirosi Ooguri, ${ }^{a}$ Andrew Strominger, ${ }^{b}$ and Cumrun Vafa ${ }^{b}$ \\ ${ }^{a}$ California Institute of Technology, Pasadena, CA 91125, USA \\ ${ }^{b}$ Jefferson Physical Laboratory, Harvard University \\ Cambridge, MA 02138, USA
}

\begin{abstract}
A simple relationship of the form $Z_{B H}=\left|Z_{\text {top }}\right|^{2}$ is conjectured, where $Z_{B H}$ is a supersymmetric partition function for a four-dimensional BPS black hole in a Calabi-Yau compactification of Type II superstring theory and $Z_{\text {top }}$ is a second-quantized topological string partition function evaluated at the attractor point in moduli space associated to the black hole charges. Evidence for the conjecture in a perturbation expansion about large graviphoton charge is given. The microcanonical ensemble of BPS black holes can be viewed as the Wigner function associated to the wavefunction defined by the topological string partition function.
\end{abstract}




\section{Contents}

1. Introduction . . . . . . . . . . . . . . . . . . . . . . . . . . . . . . . . . 1

2. Supergravity .. . . . . . . . . . . . . . . . . . . . . . . 5

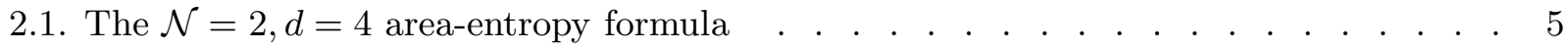

2.2. Higher order corrections . . . . . . . . . . . . . . . . . . . . . . . . . . . 7

2.3. Reinterpretation of the corrected entropy formula . . . . . . . . . . . . . . . 8

3. The topological string . . . . . . . . . . . . . . . . . . . . . . . . . . . . . 10

3.1. Black hole and topological string partition functions . . . . . . . . . . . . . . . 10

3.2. A simplified derivation? . . . . . . . . . . . . . . . . . . . . . . . . . . 12

4. The holographic CFT dual . . . . . . . . . . . . . . . . . . . . . . . . . . . 14

4.1. Gauge theory on the $D 6$ brane . . . . . . . . . . . . . . . . . . . . . . . . 14

4.2. Wrapped 5-brane dual . . . . . . . . . . . . . . . . . . . . . . . . . . . 15

4.3. The supersymmetric index . . . . . . . . . . . . . . . . . . . . . . . . . . 16

5. Counting of BPS states and topological string . . . . . . . . . . . . . . . . . . . 17

5.1. A realization of the D-brane theory for Type IIA superstrings . . . . . . . . . . 18

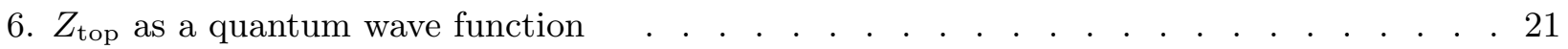

7. Further Issues . . . . . . . . . . . . . . . . . . . . . . . . . . . . . . . . . 25

7.1. Fragmentation and jumping lines . . . . . . . . . . . . . . . . . . . . . . 25

7.2. The holomorphic anomaly . . . . . . . . . . . . . . . . . . . . . . . . . . 26

7.3. Coulomb branches . . . . . . . . . . . . . . . . . . . . . . . . . 26

7.4. Hypermultiplets . . . . . . . . . . . . . . . . . . . . . . . . . . . . . . 27

\section{Introduction}

Critical string theory compactified on Calabi-Yau spaces has played a central role in both the mathematical and physical development of modern string theory. The rich topological data, as well as much of the low energy 4d physics associated with such compactifications, are elegantly summarized by the $\hat{c}=6$ topological strings of A and B types. These topological string theories have led to a variety of deep physical and mathematical insights.

The physical relevance of the data provided by the topological string has been that it computes $F$-type terms in the corresponding four dimensional theory [1,2]. These higherderivative $F$-type terms for Type II superstring on a Calabi-Yau are of the general form

$$
\int d^{4} x d^{4} \theta\left(W_{a b} W^{a b}\right)^{g} F_{g}\left(X^{\Lambda}\right),
$$

where $W_{a b}$ is the graviphoton superfield of the $\mathcal{N}=2$ supergravity and $X^{\Lambda}$ are the vector multiplet fields. The lowest component of $W$ is $F$ the graviphoton field strength and the 
highest one is the Riemann tensor. The lowest components of $X^{\Lambda}$ are the complex scalars parameterizing Calabi-Yau moduli and their highest components are the associated $U(1)$ vector fields. These terms contribute to multiple graviphoton-graviton scattering. (1.1) includes (after $\theta$ integrations) an $R^{2} F^{2 g-2}$ term. This fact was used in [3], upon considering a constant graviphoton background, to provide a precise physical interpretation of the meaning of A-model topological strings as computing degeneracy of BPS states involving M2 branes wrapped around 2-cycles of Calabi-Yau upon M-theory compactifications to five dimensions. Roughly speaking the genus expansion of topological string encodes the spin of the BPS state and the 2-cycle it wraps corresponds to the charge of the M2 brane. More precisely the topological string partition function $Z_{\text {top }}$ is the canonical ensemble for multi-particle spinning five dimensional black holes [4] as was discussed in [5].

In this paper we define and propose a simple and direct relationship between the second-quantized topological string partition function $Z_{\text {top }}$ and BPS black hole partition function $Z_{B H}$ in four dimensions of the form 1

$$
Z_{B H}\left(p^{\Lambda}, \phi^{\Lambda}\right)=\left|Z_{\text {top }}\left(X^{\Lambda}\right)\right|^{2}
$$

where $X^{\Lambda}=p^{\Lambda}+\frac{i}{\pi} \phi^{\Lambda}$ in a certain Kähler gauge. The left hand side here is evaluated as a function of integer magnetic charges $p^{\Lambda}$ and continuous electric potentials $\phi^{\Lambda}$, which are conjugate to integer electric charges $q_{\Lambda}$. The right hand side is the holomorphic square of the partition function for a gas of topological strings on a Calabi-Yau whose moduli are those associated to the charges/potentials $\left(p^{\Lambda}, \phi^{\Lambda}\right)$ via the attractor equations [6, 7, 8,9, 10, 11]. Both sides of (1.2) are defined in a perturbation expansion in 1/Q, where $Q$ is the graviphoton charge carried by the black hole. 2 The nonperturbative completion of either side of (1.2) might in principle be defined as the partition function of the holographic CFT dual to the black hole, as in [12]. We then have the triple equality,

$$
Z_{C F T}=Z_{B H}=\left|Z_{\mathrm{top}}\right|^{2}
$$

1 Here and hereafter the partition functions are presumed to contain $F^{2}(-1)^{F}$ type insertions, where $F$ is an appropriate $\mathrm{R}$ charge, so that only BPS states contribute.

2 The string coupling $g_{s}$ is in a hypermultiplet and decouples from the computation. 
This will be discussed herein for two cases: One in which the CFT is a $2 \mathrm{~d}$ sigma model arising form the moduli space of wrapped fivebranes [13] compactified on a circle, or the IR limit of the $U(N)$ gauge theory on the D6 brane considered in 14.3

As noted already in [8[10], terms such as (1.1) are nonvanishing in the presence of a black hole and therefore correct the BPS entropy. It is plausible that, as discussed in [8[10, due to supersymmetric nonrenormalization theorems, these $F$-terms are the only ones which correct the (indexed) BPS entropy. We derive (1.2) building on the supergravity analysis of [8-10]. A key new point is that the supergravity partition function naturally produces a mixed thermodynamic ensemble, in which magnetic charges $p^{\Lambda}$ are fixed integers, while electric charges $q_{\Lambda}$ are summed over weighted by the electric potential as $e^{-\phi^{\Lambda} q_{\Lambda}}$. This distinction is irrelevant to the computation of the leading order areaentropy relation. However it plays a crucial role in disentangling higher order corrections and achieving the simple relation (1.2).

The existence of fundamental connection between four-dimensional BPS black holes and the topological string might have been anticipated from the following observation. Calabi-Yau spaces have two types of moduli: Kähler and complex structure. The worldsheet twisting which produces the A (B) model topological string from the critical superstring eliminates all dependence on the complex structure (Kähler) moduli at the perturbative level. Hence the perturbative topological string depends on only half the moduli. Black hole entropy on the other hand, insofar as it is an intrinsic property of the black hole, cannot depend on any externally specified moduli (this statement is corrected by the anomaly as we discuss in the next paragraph). What happens at leading order is that the moduli in vector multiplets are driven to attractor values at the horizon which depend only on the black hole charges and not on their asymptotically specified values. Hypermultiplet vevs on the other hand are not fixed by an attractor mechanism but simply drop out of the entropy formula. Consistency suggests this mechanism should extend beyond leading order, and some evidence for this has been given in [8,9, 10]. It is natural to assume this is valid to all orders in a $1 / Q$ expansion. Hence the perturbative topological string and the large black hole partition functions depend on only half the Calabi-Yau moduli. It

3 This latter realization, even though it has the disadvantage of not being conformal, it has the advantage of being able to realize arbitrary charge states for the black hole. Having a QFT is sufficient for computation of (1.3) and the result (being an index) should not depend on taking the IR limit. 
would be surprising if string theory produced two functions on the same space that were not simply related. Indeed we shall argue that they are simply related as in (1.2).

The fact that the mixed ensemble, rather than microcanonical ensemble, should correspond to topological string is very natural. The very formulation of mixed ensemble depends on how one splits the charges to electric/magnetic pairs. Indeed topological string partition function also depends on the choice of how one splits electric and magnetic charges, as was discussed in [15]. Choice of electric versus magnetic is like a choice of position versus momentum for topological strings. In fact purely from this perspective and the commutation relations between electric and magnetic charges and the corresponding chemical potentials one can derive the fact that the imaginary part of the attractor moduli should be identified with the chemical potentials. Furthermore according to [15] $Z_{\text {top }}$ behaves as a wave function $Z_{\text {top }} \sim \psi(x)$ in a given basis and going from electric to magnetic leads to its Fourier transform $\tilde{\psi}(p)$. In this context the formula we are proposing $Z_{B H}=\left|Z_{\text {top }}\right|^{2}=|\psi|^{2}$ becomes even more suggestive and intriguing: The probability density of the topological wave function $\psi$ is being related to the number density of black holes of a fixed magenetic charge and fixed electric chemical potential. More precisely we can view the black hole microcanonical ensemble as the Wigner function on the electric/magnetic charge space determined by the topological string wave function. This is one of the most intriguing consequences of the present paper. Aspects of this relation is discussed in section 6 .

In the preceding discussion and most of the paper we ignore several important subtleties. It is known that the degeneracy of actual single particle states can jump as we change the vevs for the scalars in the vector multiplets [16]. One might try to avoid this by dropping the restriction to single black hole states in $Z_{B H}$, as we shall ultimately find appropriate. However this does not avoid the problem because the asymptotic values of the moduli still enter the index due to the noncompact center-of-mass integrations. Thus even the perturbative entropy would not be expected to depend just on the charges of the black hole, but also the background the black hole is embedded in. In fact this is consistent with another fact for topological strings which reinforces (1.2): the holomorphic anomaly! It is known that the topological string partition function $Z_{\text {top }}$ does depend on the background $\left(t_{B}, t_{B}^{*}\right)$ we expand about, and not just on the chiral vector multiplet $X$ [17,1]. In (1.2) we identify, via the attractor mechanism, the $X$ with magnetic charge and electric potential. If we identify $\left(t_{B}, t_{B}^{*}\right)$ with the background where the black hole entropy is computed in, then (1.2) is consistent with the fact that both sides do depend on 
the choice of the background. We thus expect that both sides of (1.2) to depend in addition on vector and possibly hypermultiplets of the background (the moduli at infinity) where the black hole is embedded in. Finally we note that the holographic dual partition function $Z_{C F T}$ is also likely to acquire background dependence via non-compact Coulomb branches. A partial and inconclusive discussion of these issues appear in section 7. A particualrly interesting issue is whether or not the partition functions depend on hypermultiplet vevs at the nonperturbative level via D-brane instantons as in 18, 19,20.

To summarize, we have suppressed some subtle and important issues which are ultimately essential for a complete understanding of the meaning of (1.2). Our hope is that the naive analysis presented here has suggested the correct exact relation, in which both sides of (1.2) acquire background dependence. We leave this to future investigations.

The relation (1.2) states that the supersymmetric partition function of BPS black hole microstates equals the squared partition function of a second-quantized gas of topological strings on a Calabi-Yau. Our derivation is mechanical in nature and relies on a detailed comparison of the perturbation expansion of both quantities. (We propose a streamlined derivation of this perturbative statement in section 3.3.) However we feel that such a simple physical relationship should have an equally simple physical derivation which extends beyond perturbation theory.

This paper is organized as follows: In section 2 we discuss the supergravity aspects of F-term corrections. There we motivate the relation $Z_{B H}=\left|Z_{\text {top }}\right|^{2}$ and the interpretation of $Z_{B H}$ as a mixed ensemble. In section 3 we discuss some normalization issues for relating this to topological string amplitudes and propose an alternative simple, but non-rigorous derivation of the main formula $Z_{B H}=\left|Z_{\mathrm{top}}\right|^{2}$. Section 4 discusses two choices for dual CFT's. In Section 5 we discuss the relation between our proposal with the proposed relationship between topological string amplitudes and crystal melting. Section 6 comments on the interpretation of $Z_{\text {top }}$ as a quantum amplitude. In section 7 we discuss how the entropy of black hole as well as the topological string amplitudes do depend in addition on vector multiplet background (through holomorphic anomaly) and possibly hypermultiplet background (through non-perturbative corrections).

\section{Supergravity}

\subsection{The $\mathcal{N}=2, d=4$ area-entropy formula}

In this subsection, we review the leading semiclassical area-entropy formula for a general $\mathcal{N}=2, d=4$ extremal black hole characterized by magnetic and electric 
charges $\left(p^{\Lambda}, q_{\Lambda}\right)$ derived in [6,7] and recently reviewed in [11]. The asymptotic values of the moduli in vector multiplets, parameterized by complex projective coordinates $X^{\Lambda}, \Lambda=0,1, \ldots, n_{V}$, in the black hole solution are arbitrary. These moduli couple to the electromagnetic fields and accordingly vary as a function of the radius. At the horizon they approach an attractor point whose location in the moduli space depends only on the charges. The locations of these attractor points can be found by looking for supersymmetric solutions with constant moduli. They are determined by the attractor equations, $\frac{1}{4}$

$$
\begin{aligned}
& p^{\Lambda}=\operatorname{Re}\left[C X^{\Lambda}\right], \\
& q_{\Lambda}=\operatorname{Re}\left[C F_{0 \Lambda}\right],
\end{aligned}
$$

where $F_{0 \Lambda}=\partial F_{0} / \partial X^{\Lambda}$ are the holomorphic periods, and the subscript 0 distinguishes these from the string loop corrected periods to appear in the next subsection. Both $\left(p^{\Lambda}, q_{\Lambda}\right)$ and $\left(X^{\Lambda}, F_{0 \Lambda}\right)$ transform as vectors under the $S p(2 n+2 ; Z)$ duality group.

The $\left(2 n_{v}+2\right)$ real equations (2.1) and (2.2) determine the $\left(n_{v}+2\right)$ complex quantities $\left(C, X^{\Lambda}\right)$ up to Kähler transformations, which act as

$$
K \rightarrow K-f(X)-\bar{f}(\bar{X}), \quad X^{\Lambda} \rightarrow e^{f} X^{\Lambda}, \quad F_{0} \rightarrow e^{2 f} F_{0}, \quad C \rightarrow e^{-f} C,
$$

where the Kähler potential $K$ is given by

$$
e^{-K}=i\left(\bar{X}^{\Lambda} F_{0 \Lambda}-X^{\Lambda} \bar{F}_{0 \Lambda}\right)
$$

We could at this point set $C=1$ and fix the Kähler gauge but later we shall find other gauges useful. It is easy to see that - as required - the charges $\left(p^{\Lambda}, q_{\Lambda}\right)$ determined by the attractor equations (2.1), (2.2) are invariant under Kähler transformations. Given the horizon attractor values of the moduli determined by (2.1) and (2.2) the BekensteinHawking entropy may be written

$$
S_{B H}=\frac{1}{4} \text { Area }=\pi|Q|^{2},
$$

where $Q=Q_{m}+i Q_{e}$ is a complex combination of the magnetic and electric graviphoton charges and

$$
|Q|^{2}=\frac{i}{2}\left(q_{\Lambda} \bar{C} \bar{X}^{\Lambda}-p^{\Lambda} \bar{C} \bar{F}_{0 \Lambda}\right)=\frac{C \bar{C}}{4} e^{-K}
$$

4 As in [13], we have redefined $C, X$ and $F$ by factors of $e^{K / 2}$ relative to [7] so that $\left(X^{\Lambda}, F_{\Lambda}\right)$ is a holomorphic section. At the attractor point our variables are related to those of [9] by $C X_{u s}^{L}=-2 i Y_{\text {them }}^{\Lambda}, \quad C F_{\Lambda}^{u s}\left(X^{\Lambda}\right)=-2 i F_{\Lambda}^{t h e m}\left(Y^{\Lambda}\right), \quad C=-2 i \bar{Z} e^{K / 2}$. 
The normalization of $Q$ here is chosen so that $|Q|$ equals the radius of the two sphere at the horizon.

It is useful to rephrase the above results in the context of type IIB superstrings in terms of geometry of Calabi-Yau. In this case the attractor equations fix the complex geometry of the Calabi-Yau. The electric/magentic charges correlate with three cycles of Calabi-Yau. Choosing a symplectic basis for the three cycles gives a choice of the splitting to electric and magnetic charges. Let $A_{\Lambda}$ denote a basis for the electric three cycles, $B^{\Sigma}$ the dual basis for the magnetic charges and $\Omega$ the holomorphic 3-form at the attractor point. $\Omega$ is fixed up to an overall multiplication by a complex number $\Omega \rightarrow \lambda \Omega$. There is a unique choice of $\lambda$ such that the resulting $\Omega$ has the property that

$$
p^{\Lambda}=\int_{A_{\Lambda}} \operatorname{Re} \Omega=\operatorname{Re}\left[C X^{\Lambda}\right] \quad q_{\Lambda}=\int_{B^{\Lambda}} \operatorname{Re} \Omega=\operatorname{Re}\left[C F_{0 \Lambda}\right]
$$

where $\operatorname{Re} \Omega=\frac{1}{2}(\Omega+\bar{\Omega})$. In terms of this choice the black hole entropy can be written as

$$
S_{B H}=\frac{\pi}{4} \int_{C Y} \Omega \wedge \bar{\Omega}
$$

\subsection{Higher order corrections}

In this subsection we review some results of [8,9,10,21], in which higher derivative corrections, suppressed at large charges, to the entropy5 formula 2.5 are derived from the F-term corrections to the supergravity action using Wald's method [22]. (For a comprehensive review of the results of these papers and related subjects, see [23.) All other corrections to the action involve hypermultiplets. Hypermultiplet vevs are not fixed at the horizon and can be varied continuously. If the entropy depended on hypermultiplet vevs, it could not be an intrinsic property of the black hole and the number of microstates for fixed charges could not be an integer. Hence we expect the vector multiplet $F$-terms to encode all corrections to the entropy.

$F$-term corrections to the action are encoded in a string loop corrected holomorphic prepotential

$$
F\left(X^{\Lambda}, W^{2}\right)=\sum_{h=0}^{\infty} F_{h}\left(X^{\Lambda}\right) W^{2 h}
$$

5 The term entropy in 8,9, 10,21] and here more precisely should refer to an indexed quantity protected from some types of corrections by supersymmetry. This is discussed in section 4.3 below. 
where $F_{h}$ can be computed by topological string amplitudes (as we review in the next section) and $W^{2}$ involves the square of the anti-self dual graviphoton field strength. This obeys the homogeneity equation

$$
X^{\Lambda} \partial_{\Lambda} F\left(X^{\Lambda}, W^{2}\right)+W \partial_{W} F\left(X^{\Lambda}, W^{2}\right)=2 F\left(X^{\Lambda}, W^{2}\right)
$$

Near the black hole horizon, the attractor value of $W^{2}$ obeys

$$
C^{2} W^{2}=256
$$

The exact attractor equations are then simply

$$
\begin{gathered}
p^{\Lambda}=\operatorname{Re}\left[C X^{\Lambda}\right], \\
q_{\Lambda}=\operatorname{Re}\left[C F_{\Lambda}\left(X^{\Lambda}, \frac{256}{C^{2}}\right)\right] .
\end{gathered}
$$

This is essentially the only possibility consistent with symplectic invariance. It is then argued in [8,9, 10,21] that the entropy as a function of the charges is

$$
S_{B H}=\frac{\pi i}{2}\left(q_{\Lambda} \bar{C} \bar{X}^{\Lambda}-p^{\Lambda} \bar{C} \bar{F}_{\Lambda}\right)+\frac{\pi}{2} \operatorname{Im}\left[C^{3} \partial_{C} F\right]
$$

where $F_{\Lambda}, X^{\Lambda}$ and $C$ are expressed in terms of the charges using (2.12) and (2.13).

\subsection{Reinterpretation of the corrected entropy formula}

In this subsection we reinterpret the $F$-term corrected entropy formula (2.14), and thereby cast it in a significantly simpler form.

Let us introduce $\phi^{\Lambda}$ as the imaginary parts of $C X^{\Lambda}$ and write

$$
C X^{\Lambda}=p^{\Lambda}+\frac{i}{\pi} \phi^{\Lambda}
$$

where we applied the first half of the attractor equations (2.12) for the real parts of $C X^{\Lambda}$. Defining a function $\mathcal{F}$ of $(\phi, p)$ by

$$
\begin{aligned}
\mathcal{F}(\phi, p) & =-\pi \operatorname{Im}\left[C^{2} F\left(X^{\Lambda}, \frac{256}{C^{2}}\right)\right] \\
& =-\pi \operatorname{Im}\left[F\left(C X^{\Lambda}, 256\right)\right] \\
& =-\pi \operatorname{Im}\left[F\left(p^{\Lambda}+\frac{i}{\pi} \phi^{\Lambda}, 256\right)\right],
\end{aligned}
$$


the second half of the attractor equations $(2.13)$ can be written as

$$
q_{\Lambda}=\frac{1}{2}\left(C F_{\Lambda}+\bar{C} \bar{F}_{\Lambda}\right)=-\frac{\partial}{\partial \phi^{\Lambda}} \mathcal{F}(\phi, p),
$$

where

$$
\frac{\partial}{\partial \phi^{\Lambda}}=\frac{i}{\pi C} \frac{\partial}{\partial X^{\Lambda}}-\frac{i}{\pi \bar{C}} \frac{\partial}{\partial \bar{X}^{\Lambda}}
$$

Using the homogeneity relation,

$$
C \partial_{C} F\left(X^{\Lambda}, \frac{256}{C^{2}}\right)=X^{\Lambda} \frac{\partial}{\partial X^{\Lambda}} F-2 F,
$$

and the attractor equations (2.17), the fully corrected entropy formula (2.14) is brought into the simple form

$$
S_{B H}(q, p)=\mathcal{F}(\phi, p)-\phi^{\Lambda} \frac{\partial}{\partial \phi^{\Lambda}} \mathcal{F}(\phi, p) .
$$

Here $q$ in the left-hand side and $\phi$ in the right-hand side are related by (2.17). We recognize that (2.20) combined with (2.17) is the Legendre transformation from $S_{B H}\left(q_{\Lambda}, p^{\Lambda}\right)$ to $\mathcal{F}\left(\phi^{\Lambda}, p^{\Lambda}\right)$ and that $\phi^{\Lambda}$ are chemical (electric) potentials for $q_{\Lambda}$.

In order to interpret 2.20 we note that in the usual formulation of a path integral, magnetic-type charges are associated to a choice of vector bundle and are not summed over. On the other hand electric-type charges are typically not fixed. Instead one chooses a boundary condition on the electric potential, which gives a weighted sum over electric charges. Hence the usual thermal path integral should be interpreted as a microcanonical ensemble of magnetic charges $p^{\Lambda}$ and a canonical ensemble for electric charges $q_{\Lambda}$ with potentials $\phi^{\Lambda}$. Such a "mixed" partition function can be written

$$
Z_{B H}\left(\phi^{\Lambda}, p^{\Lambda}\right)=\sum_{q_{\Lambda}} \Omega\left(p^{\Lambda}, q_{\Lambda}\right) e^{-\phi^{\Lambda} q_{\Lambda}}
$$

where $\Omega\left(p^{\Lambda}, q_{\Lambda}\right)$ are integer black hole degeneracies and $\ln \Omega\left(p^{\Lambda}, q_{\Lambda}\right)$ is the microcanonical entropy.

Hence we see that $\mathcal{F}\left(\phi^{\Lambda}, p^{\Lambda}\right)$ defined by $(2.16)$ is simply related to the mixed partition function $Z_{B H}$ as

$$
Z_{B H}\left(\phi^{\Lambda}, p^{\Lambda}\right)=\exp \left[\mathcal{F}\left(\phi^{\Lambda}, p^{\Lambda}\right)\right]
$$

and that $S_{B H}\left(q_{\Lambda}, p^{\Lambda}\right)$ computed in [8,9,10,21] based on a spacetime path integral picture is the mixed canonical/microcanonical entropy given by the Legendre transform $\mathcal{F}$ as in (2.20). We note that, according to our proposal, the microcanonical entropy $\ln \Omega\left(q_{\Lambda}, p^{\Lambda}\right)$ 
is not equal the mixed entropy $S_{B H}\left(q_{L}, p^{\Lambda}\right)$ since the Legendre transformation is not the inverse of the Laplace transformation (2.21), except in the limit of large electric charges $\left|q^{\Lambda}\right| \gg 1$, where the latter can be approximated by the former in the steepest descent method.

It is instructive to rewrite $Z_{B H}$ in terms of the complex graviphoton charge $Q=$ $Q_{m}+i Q_{e}, 6$ which is given as a function of $\phi^{\Lambda}$ and $p^{\Lambda}$ by

$$
Q^{2}=i \frac{p^{\Lambda}}{4}\left(C F_{\Lambda}-\bar{C} \bar{F}_{\Lambda}\right)+\frac{\phi^{\Lambda}}{4 \pi}\left(C F_{\Lambda}+\bar{C} \bar{F}_{\Lambda}\right)
$$

Choosing a gauge in which

$$
K=0, \quad C=2 Q, \quad W^{2}=\frac{64}{Q^{2}}
$$

at the attractor point, we find from (2.6) that

$$
C=2 Q, \quad X^{\Lambda}=\frac{p^{\Lambda}+i \phi^{\Lambda} / \pi}{2 Q}
$$

and

$$
\ln Z_{B H}=-4 \pi Q^{2} \operatorname{Im}\left[\sum_{h} F_{h}\left(\frac{p^{\Lambda}+i \phi^{\Lambda} / \pi}{2 Q}\right)\left(\frac{8}{Q}\right)^{2 h}\right] .
$$

\section{The topological string}

\subsection{Black hole and topological string partition functions}

The notion of topological string was introduced in [24]. Subsequently a connection between them and superstring was discovered: It was shown in [1,2], that the superstring loop corrected $F$-terms (2.9) can be computed as topological string amplitudes. The purpose of this subsection is to translate the supergravity notation of the previous section to the topological string notation.

The second quantized partition function for the topological string may be written

$$
Z_{\mathrm{top}}\left(t^{A}, g_{\mathrm{top}}\right)=\exp \left[F_{\mathrm{top}}\left(t^{A}, g_{\mathrm{top}}\right)\right]
$$

6 This is a slight abuse of terminology, as $Q$ is not precisely a charge. Rather it is a combination of charges and potentials. 
where

$$
F_{\text {top }}\left(t^{A}, g_{\text {top }}\right)=\sum_{h} g_{\text {top }}^{2 h-2} F_{\text {top }, h}\left(t^{A}\right)
$$

and $F_{\text {top }, h}$ is the $h$-loop topological string amplitude. The Kähler moduli are expressed in the flat coordinates

$$
t^{A}=\frac{X^{A}}{X^{0}}=\theta^{A}+i r^{A}
$$

where $r^{A}$ are the Kähler classes of the Calabi-Yau $M$ and $\theta^{A}$ are periodic $\theta^{A} \sim \theta^{A}+1$.

We would like to determine relations between supergravity quantities and topological string quantities. Using the homogeneity property (2.10) and the expansion (2.9), the holomorphic prepotential in supergravity can be expressed as

$$
\begin{aligned}
F\left(C X^{\Lambda}, 256\right) & =\left(C X^{0}\right)^{2} F\left(\frac{X^{\Lambda}}{X^{0}}, \frac{256}{\left(C X^{0}\right)^{2}}\right) \\
& =\sum_{h=0}^{\infty}\left(C X^{0}\right)^{2-2 h} f_{h}\left(t^{A}\right),
\end{aligned}
$$

where $f_{h}\left(t^{A}\right)$ is related to $F_{h}\left(X^{\Lambda}\right)$ in 2.9$)$ as

$$
f_{h}\left(t^{A}\right)=16^{2 h} F_{h}\left(\frac{X^{\Lambda}}{X^{0}}\right)
$$

This suggests an identification of the form $f_{h}\left(t^{A}\right) \sim F_{\text {top }, h}\left(t^{A}\right)$ and $g_{\text {top }} \sim\left(C X^{0}\right)^{-1}$. For later purposes, we need precise relations between supergravity and topological string quantities including numerical coefficients. These can be determined by studying the limit of a large Calabi-Yau space.

In the supergravity notation, the genus 0 and 1 terms in the large volume are given by 9

$$
\begin{aligned}
F\left(C X^{\Lambda}, 256\right) & =C^{2} D_{A B C} \frac{X^{A} X^{B} X^{C}}{X^{0}}-\frac{1}{6} c_{2 A} \frac{X^{A}}{X^{0}}+\cdots \\
& =\left(C X^{0}\right)^{2} D_{A B C} t^{A} t^{B} t^{C}-\frac{1}{6} c_{2 A} t^{A}+\cdots
\end{aligned}
$$

where

$$
c_{2 A}=\int_{M} c_{2} \wedge \alpha_{A}
$$

with $c_{2}$ being the second Chern class of $M$, and $C_{A B C}=-6 D_{A B C}$ are the four-cycle intersection numbers. These terms are normalized so that the mixed entropy $S_{B H}$ is given 
by (2.14). On the other hand, the topological string amplitude in this limit is given by 17, 1 17

$$
F_{\text {top }}=-\frac{(2 \pi)^{3} i}{g_{\text {top }}^{2}} D_{A B C} t^{A} t^{B} t^{C}-\frac{\pi i}{12} c_{2 A} t^{A}+\cdots,
$$

The normalization here is fixed by the holomorphic anomaly equations in [1], which are nonlinear equations for $F_{\text {top }, h}$. The same normalization of $F_{\text {top }}$ is also used in the M theory interpretation [3] and in the quantum foam interpretaion [25].

Comparing the one-loop terms in (3.6) and (3.8), which are independent of $g_{\text {top }}$, we find

$$
F\left(C X^{\Lambda}, 256\right)=-\frac{2 i}{\pi} F_{\text {top }}\left(t^{A}, g_{\text {top }}\right)
$$

Given this, we can compare the genus 0 terms to find

$$
g_{\mathrm{top}}= \pm \frac{4 \pi i}{C X^{0}}
$$

This implies

$$
\ln Z_{B H}=-\pi \operatorname{Im}\left[F\left(C X^{\Lambda}, 256\right)\right]=F_{\text {top }}+\bar{F}_{\text {top }} .
$$

and

$$
Z_{B H}\left(\phi^{\Lambda}, p^{\Lambda}\right)=\left|Z_{\text {top }}\left(t^{A}, g_{\text {top }}\right)\right|^{2}
$$

with

$$
t^{A}=\frac{p^{A}+i \phi^{A} / \pi}{p^{0}+i \phi^{0} / \pi}, \quad g_{\mathrm{top}}= \pm \frac{4 \pi i}{p^{0}+i \phi^{0} / \pi} .
$$

Such a simple relation between $Z_{B H}$ and $Z_{\text {top }}$ calls for an explanation. The remainder of this paper is devoted to physical interpretation of this relation.

\subsection{A simplified derivation?}

In this section we propose a simpler supergravity derivation of the relation

$$
Z_{B H}=\left|Z_{\text {top }}\right|^{2}
$$

With the exception of one assumption, which we cannot a priori justify, the proposed derivation may clarify the origin of such a relation. However we will not carefully work out the details and we omit the factors of 2 and $\pi$ in this section.

7 In [1] it was found that consistency requires that an additional factor of $1 / 2$ be multiplied to the one-loop amplitude $F_{\mathrm{top}, 1}$ defined in [17]. The factor $1 / 2$ comes from the $Z_{2}$ automorphism of the worldsheet torus. 
One main ingredient in this derivation is the observation of [26] that the $\mathcal{N}=2$ supergravity coupled to vector multiplets can be written as

$$
S=\int d^{4} x d^{4} \theta \text { (supervolume form) }+ \text { h.c. }=\int d^{4} x \sqrt{-g} R+\ldots
$$

where the supervolume form in the above depends non-trivially on curvature of the fields. This reproduces the ordinary action after integrating over $d^{4} \theta$ and picking up the $\theta^{4}$ term in the supervolume. In the context of black holes the boundary terms accompanying (3.14) give the classical black hole entropy.

We now come to the derivation of (3.13). As was observed in [1,2] topological string computes the terms

$$
F=\sum_{h=0}^{\infty} \int d^{4} x d^{4} \theta F_{h}(X)\left(W^{2}\right)^{g}+c . c .
$$

There are various terms one can get from the above action after integrating over $d^{4} \theta$. Let us concentrate on one of the terms which turns out to be the relevant one for us: Take the top components of $X^{\Lambda}$ and $W^{2}$, and absorb the $d^{4} \theta$ integral from the supervolume measure as in (3.14). We will work in the gauge $X^{0} \sim 1$ and thus $C \sim 1 / g_{\text {top }}$. As noted before in the near-horizon black hole geometry in this gauge the top component $W^{2} \sim 1 / C^{2} \sim g_{\text {top }}^{2}$ and the $X^{\Lambda}$ are fixed by the attractor mechanism. We thus have the black hole free energy

$$
\begin{aligned}
\ln Z_{B H} & =\sum_{h=0}^{\infty} g_{\mathrm{top}}^{2 h} F_{\mathrm{top}, h}\left(X^{\Lambda} / X^{0}\right) \int d^{4} x d^{4} \theta+c . c . \\
& =\sum_{g=0}^{\infty}\left(g_{\mathrm{top}}\right)^{2 h-2} F_{\mathrm{top}, h}\left(X^{\Lambda} / X^{0}\right)+c . c . \\
& =2 \operatorname{Re} F_{\mathrm{top}}
\end{aligned}
$$

where we have used the fact that $\int d^{4} x d^{4} \theta \sim 1 / g_{\mathrm{top}}^{2}$. Upon exponentiation this leads to (3.13).

Here we have shown that if we consider one absorption of $\theta^{4}$ term in (3.15) upon $d^{4} \theta$ integral we get the desired result. That there be no other terms is not obvious. For example another way to absorb the $\theta$ 's would have given the familiar term $R^{2} F^{2 g-2}$ where $F$ is the graviphoton field. However, as has been observed in [8,9.10] such terms do not contribute in the black hole background. It would be nice to find a simple way to argue why these terms do not contribute and that we are left with this simple absorption of the $\theta$ integrals. 


\section{The holographic CFT dual}

The near-horizon geometry of a four-dimensional BPS black hole is the RobinsonBertotti universe $\mathrm{AdS}_{2} \times \mathrm{S}^{2}$. On general grounds one expects a holographically dual conformally-invariant quantum mechanics $\mathrm{CFT}_{1}$, but such AdS/CFT dualities are not yet well-understand for the general case. In the special case $p^{0}=0$ a $\mathrm{CFT}_{2}$ dual is known [13], where the black hole geometry is expected to arise upon considering a specific circle compactification (i.e. a quotient of $\mathrm{AdS}_{3}$ geometry [27]). These cases are discussed in section 4.2. However to obtain the most general allowed black hole charges we could consider more general realization of the branes. In the context of type IIA superstring this can be achieved by considering the gauge theory on D6 branes wrapped on Calabi-Yau, which is a particular topolgoically twisted gauge theory studied in [14], which will be briefly reviewed in section 4.1. Since the main interest of this paper focuses on index computations the details of the CFT are not essential. The index computation can be done for the QFT even before flowing to the IR. The basic idea is that the dual QFT should involve the gauge theory living on the brane which leads to the black hole. Suppose the D brane in question has $d+1$ dimensional worldvolume. Then we write the QFT as a theory on $V^{d} \times S^{1}$ and the IR limit of this theory should lead to a dual $\mathrm{CFT}_{1}$ on $S^{1}$ (we view $R^{1}$ as the infinite radius limit of $\left.S^{1}\right)$. In such cases we would be referring to a $(d+1)$ dimensional QFT as the dual theory and sometimes we loosely call it the "CFT dual".

For the purposes of our mixed ensemble we would like to realize the theory on the brane to be such that the electric charges can be induced as excitations in the corresponding QFT. Below we will present two examples of such a theory.

\subsection{Gauge theory on the D6 brane}

Consider type IIA theory compactified on the Calabi-Yau $M$. We consider the $D 6$ and $D 4$ branes wrapped over cycles of Calabi-Yau as magnetic charged states and $D 0$ and $D 2$ branes as electrically charaged states. More specifically we wrap $N$ D6 branes on the Calabi-Yau $M$ and we get a $U(N)$ gauge theory on $M \times S^{1}$. This gauge theory is a topologically twisted version of the maximally supersymmetric Yang-Mills theory on Calabi-Yau (see [14] for details). Moreover the lower brane charges can be viewed as gauge excitations on the $D 6$ brane, as is well known. Thus we end up with a maximally supersymmetric gauge theory in 7 dimensions on $M \times S^{1}$. Fixing the $D 6$ and $D 4$ brane charges as $p^{A}$, we will denote this theory by $T\left(M, p^{A}\right)$. Here $p^{0}=N$. 
As we will discuss below we will be interested in the limit where the radius of $S^{1}$, $\beta \rightarrow 0$. In this limit the gauge theory simplifies by T-duality on the circle to a gauge thoery on $N D 5$ branes wrapping $M$. Precisely this theory was studied in [14] in finding a dual description of the topological string. We will discuss how this case is related to a special case of our conjecture in section 5 .

\subsection{Wrapped 5-brane dual}

For the other holographic dual we set one of the magnetic charges $p^{0}=0$. In this case the holographic duality can be simply understood in one higher dimension. Briefly, (see 13] for details ) it is a compactification of the $\mathrm{AdS}_{3} / \mathrm{CFT}_{2}$ duality associated to the black string obtained by wrapping fivebranes on a four-cycle in the class $p^{A}$ of a Calabi-Yau compactification of $\mathrm{M}$ theory. Membrane charge corresponds to turning on the self-dual three form flux on the 2-cycle $q_{A}$ in the fivebrane. 0-brane charge is obtained on further compactification to IIA by adding momentum to the black string wrapping the M-theory circle.

The resulting dual CFT, which we will denote $T\left(M, p^{A}\right)$ can be briefly described as follows [13]. The four-cycle is dual to an element $P$ of $H^{2}(M, Z)$, where $M$ denotes the Calabi-Yau and we assume $P$ corresponds to a very ample divisor. Then the moduli space $\mathcal{M}_{P}$ has dimension

$$
d_{P}=\frac{1}{6} \int_{M}\left(2 P^{3}+P c_{2}(M)\right)-2
$$

The theory $T\left(M, p^{A}\right)$ contains $d_{P}$ bosons with target space $\mathcal{M}_{P}$. There are also 3 more nonchiral bosons from translation in $R^{3}$. In addition reduction of the self-dual three-form living on the fivebrane gives chiral bosons. The number of such left-moving bosons is

$$
b_{2}^{-}(P)=\frac{1}{6} \int_{M}\left(4 P^{3}+5 P c_{2}(M)\right)-1
$$

while the number of right movers is

$$
b_{2}^{+}(P)=\frac{1}{6} \int_{M}\left(2 P^{3}+P c_{2}(M)\right)-1 .
$$

These massless scalars live on the signature $\left(b_{2}^{+}, b_{2}^{-}\right)$Narain lattice $\Gamma_{P}=H^{2}(P, Z)$. Membrane charges $q_{A}$ correspond to momenta in the signature $\left(1, b_{2}(M)-1\right)$ sublattice $\Gamma_{M}=H^{2}(M, Z)$ of $\Gamma_{P}$. Hence we can regard $q_{A}$ as an operator constructed from the chiral scalars in $T\left(M, p^{A}\right)$, while 0-brane charge is simply $q_{0}=L_{0}$. 
The theory $T\left(M, p^{A}\right)$ has $(0,4)$ supersymmetry, and accordingly the number of right moving fermions is

$$
N_{R}^{F}=\frac{1}{3} \int_{M}\left(2 P^{3}+P c_{2}(M)\right) .
$$

Assuming $b_{1}(M)=0$ there are no left moving fermions.

We note that a priori $T\left(M, p^{A}\right)$ depends on all the moduli of $M$ (as well as the integer magnetic charges $p^{A}$ ) through the moduli-dependence of $\mathcal{M}_{P}$. The electric charges $\left(q_{0}, q_{A}\right)$ arise as momentum operators in the theory associated the chiral scalars spanning the Narain lattice $\Gamma_{P}$.

\subsection{The supersymmetric index}

The holographic duals we have described above both contain the center of mass degree of freedom. In comparison with the $\mathrm{AdS}^{2} \times \mathrm{S}^{2}$ geometry it is natural to mod out the center of mass degree of freedom. This gets rid of an infinite volume factor as well as fermionic zero modes associated with the center of mass degree of freedom. We then define the supersymmetric index for this theory by

$$
Z_{C F T}\left(p^{A}, \phi^{\Lambda} ; \beta, M\right)=\operatorname{Tr}^{\prime}\left[(-)^{F} \exp \left(-\beta H-\phi^{\Lambda} q_{\Lambda}\right)\right]
$$

In the wrapped fivebrane description $F=2 J_{R}^{3}$. Naively one might think that $Z_{C F T}$, being an index, is invariant under all smooth deformations of $T$, in particular variations of the complex structure and Kähler moduli of $M$, and also independent of the inverse temperature $\beta$. In fact this is not obviously the case for the following reason. Consider for example the contributions with $q_{\Lambda}=0$. For the wrapped 5-brane dual, the moduli space $\mathcal{M}_{P}$ of the four cycle $P$ has points at which the single four cycle degenerates into two four-cycles. These points are junctions with Coulomb branches at which the four cycle can fragment into two four-cycles which move apart in the $R^{3}$. For the $U(N)$ gauge theory on $D 6$ brane the same phenomenon happens where there are points where $U(N) \rightarrow U(K) \times$ $U(N-k)$. Thus in either case the holographic dual theory develops a new noncompact direction and a new continuum in the spectrum.

It is well-known that the number of BPS states can jump in such a case; for example, it was observed in the case of $\mathcal{N}=2$ gauge theories in four dimensions in [16], and it is realized in the Calabi-Yau context in [28]. When this happens via the emergence of a noncompact direction, it is expected that an index becomes a continuous function of background moduli. This is shown explicitly in two-dimensional models [29, where the 
index $\operatorname{Tr}(-1)^{F} F e^{-\beta H}$ defined for Landau-Ginzburg theories turns out to be a continuous function of moduli as it receives anomalous contribution from multiparticle states, even though the number of BPS states jumps in this case.

This fragmentation phenomenon is generic [30] and occurs at many values of the charges. $\mathrm{B}$ We thus expect that $Z_{C F T}$ acquires anomalous dependence on the moduli of $M$. We will discuss this issue in more detail in secton 7. The index (4.5) can also depend on the inverse temperature $\beta$, as we will discuss in the next section. We will argue that the mixed partition function of black holes discussed in this paper is identified with the $\beta \rightarrow 0$ limit of the index.

\section{Counting of BPS states and topological string}

The aim of this section is to consider some possible checks for the conjecture $Z_{B H}=$ $\left|Z_{\text {top }}\right|^{2}$, by using the microscopic description of the black hole entropy.

Given that $Z_{B H}$ is expressed in terms of the topological string partition function as $\left|Z_{\text {top }}\right|^{2}$, it is natural to expect that we are computing an index, i.e., the number of black hole microstates of a given set of charges where we count each BPS multiplet with a \pm sign correlated with whether the lowest component is a boson or a fermion. An obvious candidate would be the index (4.5) discussed in the last section.

Due to the presence of noncompact directions, the index (4.5) may depend on $\beta$. Thus, we should ask what is the natural value for $\beta$ from the perspective of the black hole entropy. We propose that for the four-dimensional black hole under study, we should take the trace over all states of a given charge with some electric potential, regardless of their energy. In other words we should take the limit $\beta \rightarrow 0$. According to what we have found in this paper, we would then identify

$$
\lim _{\beta \rightarrow 0} Z(p, \phi ; \beta, M)=Z_{B H}=\left|Z_{\text {top }}\left(g_{\text {top }}, t ; M\right)\right|^{2}
$$

where

$$
g_{\text {top }} \sim \frac{1}{C X^{0}}, \quad t^{A} \sim \frac{X^{A}}{X^{0}}
$$

with $X$ 's are fixed by the attractor mechanism as

$$
X^{A} \sim p^{A}+i \frac{\phi^{A}}{\pi}
$$

\footnotetext{
8 A closely related phenomenon of multiple basins of attraction was pointed out in [31, 32, 33].
} 
Recently it was shown that the topological string partition function $Z_{\mathrm{top}}$ for a toric Calabi-Yau manifold is expressed in the following form [25,14,

$$
Z_{\mathrm{top}}=\sum_{q} \Omega(q) \exp \left(-g_{\mathrm{top}} q_{0}-t^{A} q_{A}\right),
$$

where $\Omega(q)$ is an integer given by the number of configuration of a classical statistical model. We will find that, when $p_{\Lambda}=0$, the limit of the black hole entropy $\lim _{\beta \rightarrow 0} Z_{B H}(p, \phi ; \beta, M)$ can also be brought into this form via a chain of duality transformations of Type II string theory. More precisely we argue that the inclusion of the anti-holomorphic contributions to the gauge theory description of [14] leads to $\left|Z_{\text {top }}\right|^{2}$ instead of $Z_{\text {top }}$. More precisely we argue that the QFT dual of our black hole in the $\beta \rightarrow 0$ and by a combination of $\mathrm{T}$ and $\mathrm{S}$ duality gets mapped to the same QFT as discussed in [14] where the transformed chemical potentials take the values given above. Thus the crystal melting ensemble is the S-dual of the mixed black hole ensemble.

\subsection{A realization of the D-brane theory for Type IIA superstrings}

Consider Type IIA superstring compactified on a Calabi-Yau threefold. In order to formulate the partition function (4.5) in the superstring context, we take a Euclidean time and compactify it on a circle of radius $\beta$ with periodic (supersymmetry preserving) boundary conditions for the fermions. We consider a system of $D 6$ and $D 4$ branes carrying magnetic charges $p^{0}$ and $p^{A}$, and regard $D 0$ and $D 2$ branes as electric excitations. The theory on the branes is a gauge theory, which is topologically twisted [34] to give the right amount of supersymmetry. Gauge theories of this type have been discussed in [35, 36]. We will consider the case with $p^{\Lambda}=0$ since this will simplify some of the analysis below. In this context we should view the gauge theory as $U(N \mid N)$ where the lower brane charges are induced as excitations of this gauge theory.

The index (4.5) can be computed either as a trace over the QFT Hilbert space (with the center-of-mass degrees of freedom removed in the case of (4.5)) or by periodically identifying the imaginary time of Euclideanized $\mathrm{AdS}_{2}$ with period $\beta$. From the latter point of view, the index is given by a sum over BPS states in $\mathrm{AdS}_{2}$. If the type IIA string coupling $g_{\mathrm{A}}$ is small, dominant contributions would come from $D 0$ and $D 2$ branes carrying electric charges $q_{0}$ and $q_{A}$ in the form,

$$
\exp \left(-\frac{\beta}{g_{\mathrm{A}}} q_{0}-\frac{\beta}{g_{\mathrm{A}}} t^{A} q_{A}\right),
$$


where $t^{A}$ is the Kähler class measured in the type IIA string frame.

Let us consider the limit $\beta \rightarrow 0$, where we expect the identification (5.1) works. In order for the $D 0$ and $D 2$ branes to give finite contributions, we also take $g_{\mathrm{A}} \rightarrow 0$ keeping the ratio $\beta / g_{\mathrm{A}} \ll 1$. Comparing this with the corresponding contribution in (4.5) from states with charges $q_{0}, q_{A}$, we can identify the type IIA variables $g_{\mathrm{A}}, t^{A}$ with the chemical potentials $\phi^{0}, \phi^{A}$ as

$$
\phi^{0}=\frac{\beta}{g_{\mathrm{A}}}, \quad \phi^{A}=\frac{\beta}{g_{\mathrm{A}}} t^{A} .
$$

The contribution of the $D 0$ branes also suggests the identification of the type IIA string coupling with the topological string coupling as

$$
g_{\mathrm{top}} \sim \frac{g_{\mathrm{A}}}{\beta} .
$$

Note that this gives an alternative derivation of the attractor mechanism. As we have shown in section $3, X^{0}, X^{A}$ are related to topological string parameters as

$$
X^{0} \sim \frac{1}{g_{\text {top }}}, \quad X^{A} \sim \frac{t^{A}}{g_{\text {top }}} .
$$

Assuming (5.4), we arrive at the relation,

$$
\begin{aligned}
& X^{0} \sim \frac{1}{g_{\mathrm{top}}} \sim \frac{\beta}{g_{\mathrm{A}}}=\phi^{0}, \\
& X^{A} \sim \frac{t^{A}}{g_{\mathrm{top}}} \sim \frac{\beta}{g_{\mathrm{A}}} t^{A}=\phi^{A} .
\end{aligned}
$$

This reproduces the attractor values (2.15) of the moduli.

Note however, that in the limit of small $\beta$ it is natural to do a $T$-duality. Taking $T$ dual around the imaginary time direction gives Type IIB theory on a circle of radius $1 / \beta$ and with string coupling $g_{\mathrm{B}}=g_{\mathrm{A}} / \beta$. As already mentioned we are interested in the limit $\beta / g_{\mathrm{A}} \ll 1$ which implies that $g_{\mathrm{B}} \gg 1$. Thus to get a better description of this type IIB theory we need to do an S-duality. After $S$ duality $g_{\mathrm{B}} \rightarrow 1 / g_{\mathrm{B}}$. Note that under S-duality $t^{I} / g_{\mathrm{B}} \rightarrow t^{I}$. By doing this S-duality the amplitudes of topological (A-model) string get related to amplitudes of $\mathrm{D}(-1)$ and $\mathrm{D} 1$ brane instantons in the $\mathrm{CY}$. This is related to the fact that topological string partition function has an expansion of the form

$$
Z_{\text {top }}=\sum_{q} \Omega(q) \exp \left(-g_{\text {top }} q_{0}-t^{A} q_{A}\right)
$$


where in the latter $q_{0}$ and $q_{A}$ can be interpreted as the D-instanton and D1-brane contribution in the type IIB theory. This result for topological string was recently derived in 25,14, for toric Calabi-Yau manifolds, where it was shown that $\Omega(q)$ counts a number of configurations in a classical statistical model describing melting of a crystal. More precisely $\Omega(q)$ is related to the partition function of the theory on a $D 5$ brane wrapping the Calabi-Yau with fixed induced $D 0$ and D2 brane charge-exactly the same theory which we have discussed as a holographic dual to the black hole. Moreover it was conjectured in [14] and in [37] that this relation is true for general compact Calabi-Yau as well. We thus find that $\Omega(q)$ is related to the number of BPS states of the black hole with $p=0$. This relation between states of the topologically twisted theory on the brane and the topological string partition function has been recently explained in [20] by using S-duality of Type IIB superstrings, which is T-dual to the S-duality we have used here. 9 The relation (5.6) is similar the relation we are anticipating from our general analysis applied to this special case. Namely the right hand side of (5.6) is similar to what we have called $Z_{B H}$ for this holographic dual. However there are two important differences: This equation relates $Z_{\text {crystal }}=Z_{\text {top }}$ instead of $Z_{B H}=\left|Z_{\text {top }}\right|^{2}$. We believe this may be due to the fact that in the analysis of [14 one was specifically considering holomorphic moduli dependence of the holographic dual Yang-Mills theory on Calabi-Yau. But there is a more fundamental difference between the two ensembles: The black hole ensemble and the crystal melting ensemble are S-dual of one another (i.e., we have a strong/weak duality on the topological string coupling constant) and so there is no one to one map from a melting crystal configuration to that of a black hole. Nevertheless, it would be important to study this relation further.

Note that the connection between topopological strings and topologically twisted Yang-Mills theory we have proposed is analogous to that of $N=2$ topological YangMills theory in 2 dimensions. In that case it is known that $N=2$ topological Yang-Mills theory is equivalent to ordinary non-supersymmetric Yang-Mills theory [39]. Moreover the ordinary Yang-Mills theory has been studied in the limit of large rank [40,41, 42, which is analogous to our semiclassical expansion of black holes, and it has been seen that it can be written as a square of a topological theory, which maps worldsheet Riemann surfaces holomorphically to the target $2 \mathrm{~d}$ space where the Yang-Mills lives on. They find for the 2D Yang-Mills case

$$
Z_{Y M}=\left|Z_{\text {top }}\right|^{2}
$$

9 See [38] for discussion of a related issue from a different point of view. 
very much in the spirit of what we are predicting for the higher dimensional topologically twisted version of YM on CY.

Let us now turn to the other limit $\beta \rightarrow \infty$. In this case, only ground states can contribute to (4.5). In order for $D 0$ and $D 2$ branes contribute appreciably, as is evident from (4.5), we need to take $g_{\mathrm{A}} \rightarrow \infty$ so that $g_{\mathrm{A}} / \beta$ stays finite. Thus, it is natural that the $\lim _{\beta \rightarrow \infty} Z$ is related to a $5 \mathrm{~d}$ black hole computation in $\mathrm{M}$ theory on the Calabi-Yau space times a circle. This is in fact the duality explored in [3] where M2 brane degeneracies was connected to topological A-model strings. More precisely in this five-dimensional context one identifies the $t^{A}$ chemical potential, as the chemical potential for the $M 2$ brane, and the $g_{\text {top }}$ with the chemical potential for the $J_{3}$ generator in $S U(2)_{L}$ subgroup of the $S U(2)_{L} \times S U(2)_{R}=S O(4)$ spatial rotation group in five dimensions.

It is natural also to ask what does the $\beta$ dependence of $Z$ mean for the black hole entropy. It is morally a black hole in the background of "supersymmetric temperature $\frac{1}{\beta}$ " (due to periodic fermionic boundary conditions).

\section{6. $Z_{\text {top }}$ as a quantum wave function}

The relation $Z_{B H}=\left|Z_{\text {top }}\right|^{2}$ suggests that $Z_{\text {top }}$ should be interpreted as a quantum state and $Z_{B H}$ the corresponding probability amplitude. Roughly speaking, $Z_{\text {top }}$ is the quantum amplitude for finding a black hole with charges $\left(p^{\Lambda}, q_{\Lambda}\right)=\left(\operatorname{Re} C X^{\Lambda}, \operatorname{Re} C F_{\Lambda}\right)$.

This fits nicely with the discussion in [15], where it was observed that the holomorphic anomaly of [1] can be interpreted in a simple way if one identifies $Z_{\text {top }}$ as a state in an "auxiliary" Hilbert space arising from geometric quantization of the moduli space $\mathcal{M}_{M}$ of the Calabi-Yau $M$ as a phase space: In the B-model context we define

$$
C X^{\Lambda}=\int_{A_{\Lambda}} \Omega, \quad C F_{\Lambda}=\int_{B^{\Lambda}} \Omega
$$

where $A_{\Lambda}, B^{\Sigma}$ are symplectic conjugate 3 -cycles and assign a commutation relation which in our normalizations is given by

$$
\left[C X^{\Lambda}, C F_{\Sigma}\right]=\frac{2 i}{\pi} \delta_{\Sigma}^{\Lambda}
$$

or equivalently

$$
\left[X^{\Lambda}, F_{\Sigma}\right]=\frac{2 i}{\pi C^{2}} \delta_{\Sigma}^{\Lambda}
$$


The topological string amplitudes depend only on half of these variables, which we take to by $X^{\Lambda}$. The choice of the background Calabi-Yau $M_{0}$ which one expands about changes what one means by $\Omega$ and thus how one writes the $Z_{\text {top }}\left(X^{\Lambda}\right)$. This dependence on $M_{0}$ was proposed in [15] to be equivalent to the holomorphic anomaly equation of [1]. This relation has been further elucidated recently in [43]. Note that $Z_{\text {top }}$ not only depends on the background (whose black hole interpretation we will discuss in the next section) but it also depends on the choice of a symplectic basis of 3-cycles (i.e. the separation of 3-cycles into the A and B cycles). In this section we would like to explore, for a fixed background $M_{0}$ the dependence of $Z_{\text {top }}$ on the choice of the symplectic basis.

Note that (6.1) is compatible with the semiclassical statement that $F_{\Sigma}=\partial_{\Sigma} F_{0}\left(X^{\Lambda}\right)$. To see this recall that in our normalization we have

$$
\begin{aligned}
e^{F_{\mathrm{top}}} & =\exp \left[\frac{\pi i}{2} C^{2} F\left(X, \frac{256}{C^{2}}\right)\right] \\
& =\exp \left[\frac{\pi i}{2} C^{2} F_{0}(X)+\cdots\right]
\end{aligned}
$$

Note that $1 / C$ plays the role of the topological string coupling constant $g_{\text {top }}$. Thus, representing

$$
F_{\Sigma}=-i \frac{2}{\pi C^{2}} \frac{\partial}{\partial X^{\Sigma}}
$$

acting on the topological string wave function and taking the semi-classical limit $C \rightarrow \infty$ (i.e., $g_{\text {top }} \rightarrow 0$ ), we recover the classical relation $F_{\Sigma}=\partial_{\Sigma} F_{0}\left(X^{\Lambda}\right)$.

In our context we also have to deal with the anti-topological sector. The same analysis goes through. To keeps the sign conventions compatible with our previous discussion note that

$$
e^{\bar{F}_{\mathrm{top}}}=\exp \left[-i \frac{\pi}{2} \bar{C}^{2} \bar{F}_{0}\left(\bar{X}^{\Lambda}\right)+\cdots\right]
$$

which is compatible with the commutation relation

$$
\left[\bar{X}^{\Lambda}, \bar{F}_{\Sigma}\right]=-\frac{2 i}{\pi \bar{C}^{2}} \delta_{\Sigma}^{\Lambda}
$$

Moreover the topological and anti-topological amplitudes are independent of one another, which implies that all the barred variables commute with all the unbarred variables.

How are these commutation relations compatible with our black hole interpretation? To see this recall

$$
\begin{aligned}
& p^{\Lambda}=\operatorname{Re}\left[C X^{\Lambda}\right] \\
& q_{\Lambda}=\operatorname{Re}\left[C F_{\Lambda}\right]
\end{aligned}
$$


Using the above commutation relations we learn that, as expected the electric and magnetic charges do commute:

$$
\left[p^{\Lambda}, q_{\Sigma}\right]=0 .
$$

This is expected because we can measure both at the same time. However, we can learn something new from these commutation relations: So far we have talked about electric chemical potentials $\phi^{\Lambda}$. We could also define magnetic chemical potentials $\chi_{\Lambda}$. Note that because of their definition one expects these to be conjugate to the corresponding charges (this is true if we replace sum over charges by integrals which is true to all order in string expansion)

$$
\begin{array}{cc}
{\left[\phi^{\Lambda}, q_{\Sigma}\right]=\delta_{\Sigma}^{\Lambda}} & {\left[\chi_{\Sigma}, p^{\Lambda}\right]=\delta_{\Sigma}^{\Lambda}} \\
{\left[\phi^{\Lambda}, p^{\Sigma}\right]=0} & {\left[\chi_{\Sigma}, q_{\Lambda}\right]=0}
\end{array}
$$

For these to be compatible with the above commutation relation we are led to the unique choice

$$
\begin{aligned}
& C X^{\Lambda}=p^{\Lambda}+\frac{i}{\pi} \phi^{\Lambda} \\
& C F_{\Lambda}=q_{L}+\frac{i}{\pi} \chi_{\Lambda},
\end{aligned}
$$

which is exactly the relations we had obtained before. We thus see that the structure of topological string anticipates the identification of chemical potential with the imaginary parts of the periods.

Now we come to another issue which is the dependence of $Z_{\text {top }}$ on the choice of the symplectic basis. The fact that $Z_{\mathrm{top}}$ does depend non-trivially on a choice of symplectic basis is perfectly compatible with the black hole interpretation of it: The choice of the mixed ensemble also depends on which charges we choose as electric and which ones we choose as magnetic. However topological string amplitudes tells us how the wave function $Z_{\text {top }}$ changes as we change this basis. For example if we switch the role of $A$ and $B$ cycles, i.e. exchange electric and magnetic charges, the topological string partition function goes to its Fourier transform. What does this mean for our black hole interpretation?

To gain insight into this let us turn off the chemical potentials. So we have $X^{\Lambda} \sim$ $p^{\Lambda}, F_{\Sigma} \sim q_{\Sigma}$. Let us denote

$$
\begin{aligned}
& Z_{\text {top }}\left(X^{\Lambda}\right)=Z_{\text {top }}\left(p^{\Lambda}\right)=\tilde{\psi}(p) \\
& Z_{\text {top }}\left(F_{\Sigma}\right)=Z_{\text {top }}\left(q_{\Sigma}\right)=\psi(q)
\end{aligned}
$$


The fact that exchanging A and B cycles conjugates the variables implies that $\psi(q)$ and $\tilde{\psi}(p)$ are Fourier transform of one another. In other words

$$
\psi(q)=\int d p \tilde{\psi}(p) \exp \left(2 \pi i p^{\Lambda} q_{\Lambda}\right)
$$

(note that this expression is well defined in the perturbative topological string expansion where $q, p$ are not fixed to be integer).

Let us relate these to black hole partition function. When we set chemical potentials to zero we have

$$
\begin{aligned}
& Z_{B H}=\sum_{q} \Omega(p, q)=|\tilde{\psi}(p)|^{2} \\
& Z_{B H}=\sum_{p} \Omega(p, q)=|\psi(q)|^{2}
\end{aligned}
$$

We can invert this relation as well. Namely, if we are given the topologial string amplitude $\psi(q)$ we could ask how does this lead to $\Omega(p, q)$ ? This can be done by inverting the relation $Z_{B H}=\left|Z_{\text {top }}\right|^{2}$ and we find (ignoring for simplicity the discretization of charge, which is fine to all orders in perturbative string expansion)

$$
\Omega(p, q)=\int d \chi \exp (-p \chi)\left|\psi\left(q+i \frac{\chi}{\pi}\right)\right|^{2}
$$

The fact that the topological string amplitude transforms as a wavefunction, i.e. that $\phi(p)$ is Fourier transform of $\psi(q)$ implies that even if we had chosen a different polarization we would have gotten the same answer, in other words we can also write

$$
\Omega(p, q)=\int d \phi \exp (-q \phi)\left|\tilde{\psi}\left(p+\frac{i}{\pi} \phi\right)\right|^{2}
$$

Note that this relation between $\Omega$ and $\psi$ or $\phi$ is a bit formal, and to makes sense of it we have to choose a suitable contour. In fact this is the reason why $\Omega$ does not a priori have to be positive. It is natural to choose the contour so it looks like a Fourier transform and we have

$$
\Omega(p, q)=\int d \chi \exp (-i p \chi) \psi\left(q+\frac{\chi}{\pi}\right) \psi^{*}\left(q-\frac{\chi}{\pi}\right)
$$

Note that this relation implies that $\Omega$ is real, but it is not necessarily positive. We recognize $\Omega$ as the Wigner function associated to the wave function $\psi$. This is familiar in the reformulation of quantum mechanics as a deformation quantization (see 444 for a review and further references). In the context of this reformulation of quantum mechanics the 
fact that Wigner's function is a 'quasi-probability distribution' refers to the fact that $\Omega$ need not be positive. For us this is also the case because $\Omega$ is an index of the black hole. Even though the lack of positivity was a difficult thing to interpret in the context of Wigner's reformulation of quantum mechanics, it is perfectly natural for us in the context of the relation between the black hole and topological strings: The black hole entropy is an indexed object.

These formulae are very suggestive and in need of a deeper physical explanation: Is black hole teaching us about a reformulation of quantum mechanics? Are the black hole information puzzles resolved in this new reformulation?

\section{Further Issues}

In most of the preceding sections we have ignored important subtleties related to background dependence. These subtleties arise in all three pictures. In the supergravity picture we have black hole fragmentation, in the dual CFT picture we have multiple Coulomb branches and in the topological string picture we have the holomorphic anomaly. Although we have not understood these issues in detail, we believe that they are different manifestations of the same thing, and that a relation of the form $Z_{C F T}=Z_{B H}=\left|Z_{\text {top }}\right|^{2}$ will survive these corrections. This section contains some preliminary discussion of these issues.

\subsection{Fragmentation and jumping lines}

Usually it is assumed that the number of BPS states of a single black hole depends only on the charges of the black hole. However this is not quite true for the case of $4 \mathrm{~d}$ BPS black holes obtained from compactification of Type II superstrings on Calabi-Yau threefolds. There are two main issues. The first one is that it is known that the number of BPS states preserving half of the $\mathcal{N}=2$ supersymmetries does not depend just on the charges; it was already discovered in [16] that the number of such states can jump as we change the moduli of the scalars in the vector multiplet. This is likely related to the black hole fragmentation discussed in [30] as well as the phenomenon of multiple basins of attraction [31,32,33. We thus expect $Z_{B H}$ not only to depend on $X$, which captures the magnetic charge and the electric potentials, but also on the "background", i.e. the asymptotic value of the moduli in the supergravity solution. The asymptotic 
vector multiplet background is denoted by $t_{V}$. (Possible hypermultiplet dependence is also discussed below.)

Next we ask whether or not $t_{V}$ dependence appears in the supersymmetric index,

$$
\operatorname{Tr}^{\prime}\left[(-)^{F} e^{-\phi^{\Lambda} q_{\Lambda}}\right]
$$

In doing so we must specify whether the trace is over multi-black hole or single black hole states. The spectrum of single black hole states changes erratically as the asymptotic moduli cross basins of attraction or jumping lines. Hence the trace will be a discontinuous function of the background moduli. The other option is to include multi-black-hole states in the trace. However in that case the spectrum will in general have many continuous parameters arising from the non-compact $R^{3}$ positions of the black holes (which are nevertheless expected to yield finite answers due to the index nature of the computation). The index can still be defined in this context, but it will depend on the asymptotic values of the moduli. Hence either way we have background dependence. The best we can do is to define the multi-black hole index which has a smooth background dependence:

$$
Z_{B H}=Z_{B H}\left(p^{\Lambda}, \phi^{\Lambda} ; t_{V}\right)
$$

\subsection{The holomorphic anomaly}

Now we come to the discussion of similar issues for the topological strings. It is known that the perturbative topological string partition function depends on the background they are expanded about. This is encoded in the holomorphic anomaly of topological strings [1,17]. Thus, at least perturbatively we know that

$$
Z_{\mathrm{top}}^{\text {pert. }}=Z_{\mathrm{top}}^{\text {pert. }}\left(X^{\Lambda} ; t_{V}\right)
$$

This fits with the expectation on $Z_{B H}$ in the above. An important check would be to show that the $t_{V}$ dependence in $(7.2)$ agrees with that of $(7.3)$.

\subsection{Coulomb branches}

In the dual CFT picture we have the graded partition function $Z_{C F T}$ of fivebranes with fluxes wrapping Calabi-Yau four-cycles, or D6 branes wrapping the Calabi-Yau. At certain points of their moduli space, the fivebranes/D6 branes can fragment and move apart in $R^{3}$. This again leads to dependence on $t_{V}$. 


\subsection{Hypermultiplets}

The dependence on background vector multiplets is however not the end of the story; it is also known that the number of BPS states do change even as we change the hypermultiplet moduli. To give a simple example, consider Type IIA strings compactified on the

quintic threefold. Suppose we wish to know the number of BPS D2 branes wrapping the basic cycle of quintic once. It is known that the number of such D2 branes does depend on the complex structure of the quintic. The complex structure in this case is part of the hypermultiplet moduli. Thus we expect that the number of BPS states to also depend on the background hypermultiplet moduli, which we denote by $t_{H}$. In other words it is already known that we should expect the relevant black hole partition function to be a function of $t_{H}$. However it is not clear whether or not this $t_{H}$ dependence persists when we consider the index. Naively one may think it disappears.

Some evidence that $t_{H}$ dependence may indeed persist is that there exist natural nonperturbative completions of the topological string which depend on $t_{H}$. This is for example natural in the twistor application of topological strings [18] where the D-instantons of the topological string play a role. As noted in [19,20] these D-branes couple to $t_{H}$, and thus we expect that the full non-perturbative topological string to also depend on it, in other words one might expect non-perturbatively

$$
Z_{\text {top }}\left(X^{\Lambda} ; t_{V}, t_{H}\right)
$$

If so it would be natural to expect that our relation continues to hold even after we take into account these subtleties on both sides, taking the form

$$
Z_{B H}\left(X^{\Lambda} ; t_{V}, t_{H}\right)=\left|Z_{\text {top }}\left(X^{\Lambda} ; t_{V}, t_{H}\right)\right|^{2}
$$

Consistency of this relation with $t_{H}$-independence of perturbative topological string would require that the dependence of the black hole entropy on hypermultiplets is not present to all orders in the $(1 / Q)$ expansion, and only shows up in non-perturbative terms of the form $\exp (-Q)$.

It is also conceivable that there are other completions of topological strings which do not depend on $t_{H}$. This would in particular require setting the D-brane contributions to zero by hand, as they clearly do couple to $t_{H}$. If there were such alternative nonperturbative definitions we could imagine having

$$
Z_{B H}^{\text {index }}\left(X^{\Lambda} ; t_{V}\right)=\left|Z_{\text {top }}\left(X^{\Lambda}, t_{V}\right)\right|^{2} .
$$


The question is analogous to asking "can we define alternative definitions of topological Type II superstrings in which D-branes decouple?" One might think this is unlikely and that the $t_{H}$ dependence of $Z_{B H}$ should be there. As is clear we have just opened up a direction of investigation. Much more work is needed for a deeper understanding of the beautiful link between topological strings and black holes.

\section{Acknowledgements}

We would like to thank M. Aganagic, F. Denef, R. Dijkgraaf, M. Douglas, G. Moore, A. Neitzke, M. Rocek, A. van Proeyen and E. Witten for valuable discussions. The work of HO was supported in part by DOE grant DE-FG03-92-ER40701, that of AS was supported in part by DOE grant DE-FG02-96ER and that of CV was supported in part by NSF grants PHY-0244821 and DMS-0244464. 


\section{References}

[1] M. Bershadsky, S. Ceccoti, H. Ooguri and C. Vafa, "Kodaira-Spencer theory of gravity and exact results for quantum string amplitudes," Commun. Math. Phys. 165, 311 (1994), hep-th/9309140.

[2] I. Antoniadis, E. Gava, K. S. Narain and T. R. Taylor, "Topological amplitudes in string theory," Nucl. Phys. B 413, 162 (1994), hep-th/9307158.

[3] R. Gopakumar and C. Vafa, "M-theory and topological strings, I,II," hep-th/9809187, hep-th/9812127.

[4] J. C. Breckenridge, R. C. Myers, A. W. Peet and C. Vafa, "D-branes and spinning black holes," Phys. Lett. B 391, 93 (1997), hep-th/9602065.

[5] S. Katz, A. Klemm and C. Vafa, "M-theory, topological strings and spinning black holes," Adv. Theor. Math. Phys. 3, 1445 (1999), hep-th/9910181;

T. J. Hollowood, A. Iqbal and C. Vafa, "Matrix models, geometric engineering and elliptic genera," hep-th/0310272.

[6] S. Ferrara, R. Kallosh and A. Strominger, "N $\mathcal{N}=2$ extremal black holes", Phys. Rev. D52, 512 (1995), hep-th/9508072.

[7] A. Strominger, "Macroscopic Entropy of $\mathcal{N}=2$ black holes," Phys. Lett. B383 (1996) 39 , hep-th/9602111.

[8] G. Lopes Cardoso, B. de Wit and T. Mohaupt, "Corrections to macroscopic supersymmetric black-hole entropy," Phys. Lett. B 451, 309 (1999), hep-th/9812082.

[9] G. Lopes Cardoso, B. de Wit and T. Mohaupt, "Deviations from the area law for supersymmetric black holes," Fortsch. Phys. 48, 49 (2000), hep-th/9904005.

[10] G. Lopes Cardoso, B. de Wit and T. Mohaupt, "Macroscopic entropy formulae and non-holomorphic corrections for supersymmetric black holes," Nucl. Phys. B 567, 87 (2000), hep-th/9906094.

[11] G. W. Moore, "Les Houches lectures on strings and arithmetic," hep-th/0401049.

[12] A. Strominger and C. Vafa, "On the microscopic origin of the Bekenstein-Hawking entropy," Phys. Lett. B379 (1996) 99, hep-th/9601029.

[13] J. M. Maldacena, A. Strominger and E. Witten, "Black hole entropy in M-theory," JHEP 9712, 002 (1997), hep-th/9711053.

[14] A. Iqbal, N. Nekrasov, A. Okounkov and C. Vafa, "Quantum foam and topological strings," hep-th/0312022.

[15] E. Witten, "Quantum background independence in string theory," hep-th/9306122.

[16] N. Seiberg and E. Witten, "Electric-magnetic duality, monopole condensation, and confinement in $\mathcal{N}=2$ supersymmetric Yang-Mills theory," Nucl. Phys. B 426, 19 (1994) [Erratum-ibid. B 430, 485 (1994)], hep-th/9407087.

[17] M. Bershadsky, S. Cecotti, H. Ooguri and C. Vafa, "Holomorphic anomalies in topological field theories," Nucl. Phys. B 405, 279 (1993), hep-th/9302103. 
[18] E. Witten, "Perturbative gauge theory as a string theory in twistor space," hepth/0312171.

[19] A. Neitzke and C. Vafa, " $\mathcal{N}=2$ strings and the twistorial Calabi-Yau," hepth/0402128.

[20] N. Nekrasov, H. Ooguri and C. Vafa, "S-duality and topological strings," hepth/0403167.

[21] G. Lopes Cardoso, B. de Wit and T. Mohaupt, "Area law corrections from state counting and supergravity," Class. Quant. Grav. 17, 1007 (2000), hep-th/9910179.

[22] R. M. Wald, "Black hole entropy in the Noether charge," Phys. Rev. D 48, 3427 (1993), gr-qc/9307038;

V. Iyer and R. M. Wald, "Some properties of Noether charge and a proposal for dynamical black hole entropy," Phys. Rev. D 50, 846 (1994), gr-qc/9403028.

[23] T. Mohaupt, "Black hole entropy, special geometry and strings," Fortsch. Phys. 49, 3 (2001), hep-th/0007195.

[24] E. Witten, "On the structure of the topological phase of two-dimensional gravity," Nucl. Phys. B 340, 281 (1990).

[25] A. Okounkov, N. Reshetikhin and C. Vafa, "Quantum Calabi-Yau and classical crystals," hep-th/0309208.

[26] E. Sokatchev, "A superspace action for $\mathcal{N}=2$ supergravity," Phys. Lett. B 100, 466 (1981).

[27] A. Strominger, "AdS 2 quantum gravity and string theory," JHEP 9901, 007 (1999) hep-th/9809027.

[28] S. Kachru, A. Klemm, W. Lerche, P. Mayr and C. Vafa, 'Nonperturbative results on the point particle limit of $\mathcal{N}=2$ heterotic string compactifications," Nucl. Phys. B 459, 537 (1996), hep-th/9508155;

A. Klemm, W. Lerche, P. Mayr, C. Vafa and N. P. Warner, "Self-dual strings and $\mathcal{N}=2$ supersymmetric field theory," Nucl. Phys. B 477, 746 (1996), hep-th/9604034.

[29] S. Cecotti, P. Fendley, K. A. Intriligator and C. Vafa, "A new supersymmetric index," Nucl. Phys. B 386, 405 (1992), hep-th/9204102.

[30] J. M. Maldacena, J. Michelson and A. Strominger, "Anti-de Sitter fragmentation," JHEP 9902, 011 (1999), hep-th/9812073.

[31] G. W. Moore, "Arithmetic and attractors," hep-th/9807087.

[32] G. W. Moore, "Attractors and arithmetic," hep-th/9807056.

[33] F. Denef, "Supergravity flows and D-brane stability," JHEP 0008, 050 (2000), hepth/0005049.

[34] M. Bershadsky, C. Vafa and V. Sadov, "D-branes and topological field theories," Nucl. Phys. B 463, 420 (1996), hep-th/9511222.

[35] L. Baulieu, H. Kanno and I. M. Singer, "Special quantum field theories in eight and other dimensions," Commun. Math. Phys. 194, 149 (1998), hep-th/9704167; 
B. S. Acharya, M. O'Loughlin and B. Spence, "Higher-dimensional analogues of Donaldson-Witten theory," Nucl. Phys. B 503, 657 (1997), hep-th/9705138;

M. Blau and G. Thompson, "Euclidean SYM theories by time reduction and special holonomy manifolds," Phys. Lett. B 415, 242 (1997), hep-th/9706225.

[36] S. Donaldson and R. Thomas, "Gauge theory in higher dimensions," in The geometric universe; science, geometry, and the work of Roger Penrose, S. Huggett et. al eds., Oxford Univ. Press. 1998.

[37] D. Maulik, N. Nekrasov, A. Okounkov and R. Pandharipande, "Gromov-Witten theory and Donaldson-Thomas theory," math.AG/0312059.

[38] A. Kapustin, "Gauge theory, topological strings, and S-duality," hep-th/0404041.

[39] E. Witten, "Two-dimensional gauge theories revisited," J. Geom. Phys. 9, 303 (1992), hep-th/9204083.

[40] D. J. Gross, "Two-dimensional QCD as a string theory," Nucl. Phys. B 400, 161 (1993), hep-th/9212149.

[41] D. J. Gross and W. I. Taylor, "Two-dimensional QCD is a string theory," Nucl. Phys. B 400, 181 (1993), hep-th/9301068; "Twists and Wilson loops in the string theory of two-dimensional QCD," Nucl. Phys. B 403, 395 (1993), hep-th/9303046.

[42] S. Cordes, G. W. Moore and S. Ramgoolam, "Large $N$ 2-D Yang-Mills theory and topological string theory," Commun. Math. Phys. 185, 543 (1997), hep-th/9402107.

[43] R. Dijkgraaf, E. Verlinde and M. Vonk, "On the partition sum of the NS five-brane," hep-th/0205281.

[44] C. K. Zachos, "Deformation quantization: Quantum mechanics lives and works in phase-space," Int. J. Mod. Phys. A 17, 297 (2002), hep-th/0110114. 